UVX 2010 (2011) 197-201

DOI: $10.1051 / \mathrm{uvx} / 2011028$

(C) Owned by the authors, published by EDP Sciences, 2011

\title{
$\mathrm{SiO}_{2}$ thin-film nanopatterning by UV laser illumination of self-assembled microsphere monolayers
}

\author{
D. Grojo ${ }^{1}$, L. Charmasson ${ }^{1}$, A. Pereira ${ }^{2}$, M. Sentis ${ }^{1}$ and Ph. Delaporte ${ }^{1}$ \\ ${ }^{1}$ LP3-UMR 6182 CNRS/Aix-Marseille Université, Marseille, France \\ ${ }^{2}$ LPCML-UMR5620 CNRS/Université Lyon 1, Villeurbanne, France
}

\begin{abstract}
By illuminating transparent microsphere monolayers with single UV nanosecond laser pulses, we produce arrays of nanopatterns in oxide film on silicon. The reported features reveal the nature of the interaction of the photonic nanojets inside the oxide layer and at the highly absorbing substrate surface depending on the film thickness and the laser pulse energy.
\end{abstract}

\section{INTRODUCTION AND METHOD}

Due to downscaling of integrated devices, it is necessary to continuously improve lithographic and laser writing fabrication technologies. There are two optical methods that can reach patterning at the nanometer scale: (1) first is to lower the diffraction limit by shortening the wavelength to its minimum. Using this today approach, the fabrication of ultrahigh-density devices routinely uses deep UV illuminations [1]. (2) Alternatively, one can also convert propagative waves to highly localized light energy by near field scattering techniques [2]. Then the diffraction limit does not apply and optical fabrication methods can be performed with outstanding spatial control. In this paper, we combine both approaches by inducing direct laser writing with UV light at the vicinity of microspheres - that is in a near-field scattering domain. While the method is still far to be optimized it opens new routes to produce nanodevices.

Our work relies on recent studies based on a simple method using laser light interaction with small spherical microparticles $(R=100 \mathrm{~nm}-10 \mu \mathrm{m})$ to produce so-called photonic nanojets at the surface of any substrate supporting the spheres. When modest energy laser pulses are used, only locations of the substrate at the touch of the photonic nanojets are modified [3-6]. Taking benefit of the selfassembly properties of microspheres, this way is used to produce directly a well-ordered array of nanobeams leaving behind periodic features with size on the order of $100 \mathrm{~nm}$, depending on the material response [7-9]. As model systems, we prepare colloid array assemblies on oxidized Si substrates (fig. 1(a)). These are illuminated with UV nanosecond laser light. By gradually increasing the thickness of the oxide layer from $100 \mathrm{~nm}$ to $1 \mu \mathrm{m}$, we take away the Si highly absorbing substrate from the light nanojets. Looking at the produced features, it reveals the magnitude and the geometrical characteristics of local fields. These are compared with finite-difference time domain (FDTD) calculations of the electric fields in the structures (fig. 1(c)). More generally, the approach provides a way to control and to understand the fundamental aspects associated with material processing by near-field enhancements.

This is an Open Access article distributed under the terms of the Creative Commons Attribution-Noncommercial License 3.0, which permits unrestricted use, distribution, and reproduction in any noncommercial medium, provided the original work is properly cited. 


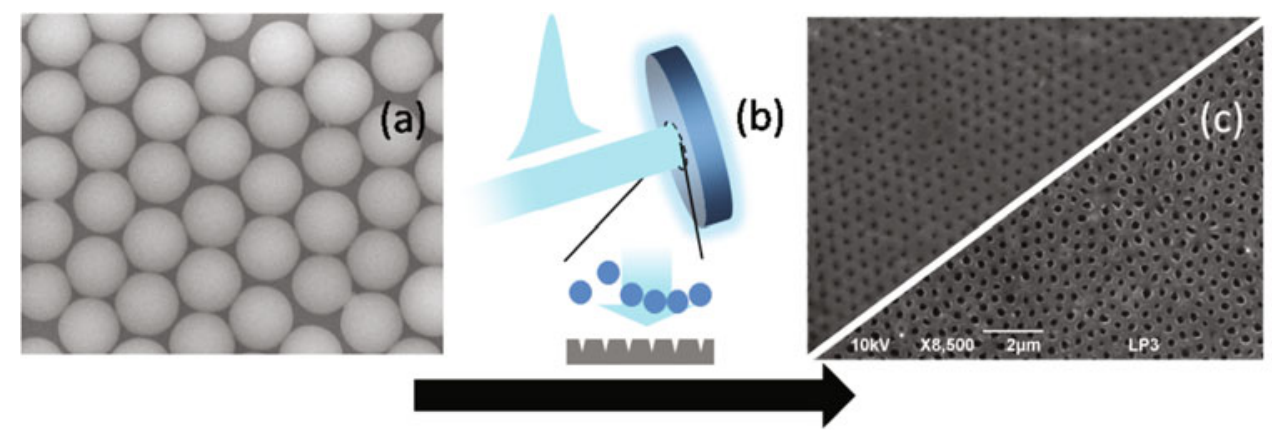

Figure 1. Method: (a) a self-assembled microsphere monolayer is prepared on top of a surface; (b) the substrate is subsequently illuminated by a single UV laser pulse at moderate energy level. Local ablation ejects the spheres leaving behind a periodically nanopatterned surface (c). (c) are SEM top-view images of a $100 \mathrm{~nm}$ oxide film after illumination with a laser fluence of $F=700 \mathrm{mJcm}^{-2}$ (top) and $F=1000 \mathrm{mJcm}^{-2}$ (bottom).

\section{EXPERIMENTAL DETAILS}

\subsection{Sample preparation and analysis}

Among the numerous self-assembly strategies available [10], we choose the dip-coating technique to produce monolayers of assembled spheres. For most experiments, we use a commercial solution (GKisker Biotech) of monodisperse silica $\left(\mathrm{SiO}_{2}\right)$ spheres with diameter $d=500 \mathrm{~nm}$. The solution is first diluted in $\approx 8 \mathrm{~mL}$ of ethanol $\left(40 \mu \mathrm{L}\right.$ at $\left.50 \mathrm{mgmL}^{-1}\right)$. Then, it is placed in a cell with the substrate making an angle of $\sim 85^{\circ}$ from horizontal. The solution is left 8 hours at $60^{\circ} \mathrm{C}$ to achieve a controlled evaporation of the solvent and a progressive colloidal self-organization on the substrate surface driven by capillary forces $[18,19]$. We have shown in previous works that residual water molecules trapped in nano-interstices affect severely light induced material detachment [13]. To avoid these effects, the substrates are maintained during few additional hours at $160^{\circ} \mathrm{C}$ so that residual humidity evaporates before their use in the experiments. After laser exposure, the samples are observed by optical and scanning electron microscopy (SEM, JEOL JSM-6390).

\subsection{Laser experiments}

In the experiments, we use an ArF nanosecond laser system (Lambda Physik, LP220i) that delivers laser pulses of $\approx 15 \mathrm{~ns}$ duration and $193 \mathrm{~nm}$ wavelength. A metallic mask was imaged using a lens of 100 $\mathrm{mm}$ focal length. The image relay technique permits a near-uniform irradiation $\left(S \cong 100 \times 100 \mathrm{~mm}^{2}\right)$ of the target materials. The laser-pulse energy is varied with the aid of a manually operated beam attenuator (Optec, AT4030). Before substrate illumination, pulse energy is measured using an energy meter (Gentec). The spatial profile of the beam is nearly flat-top so the reported laser fluences are simply expressed as $F=E / \mathrm{S}$, where $E$ is the pulse energy and $S$ the laser beam area at the target. All experiments are performed in air at atmospheric pressure. The target sample is mounted onto an $\mathrm{x}-\mathrm{y}$ translational stage. Between laser shots, the pulse energy is adjusted and the sample is moved so there is no overlap between the irradiated regions. The experiment is repeated to perform illuminations for gradually increasing fluences.

\subsection{Simulations}

To predict the near-field enhancements, we use a finite-difference time domain (FDTD) program (Lumerical). To avoid complex calculations, the numerical simulation are performed on single particles. 

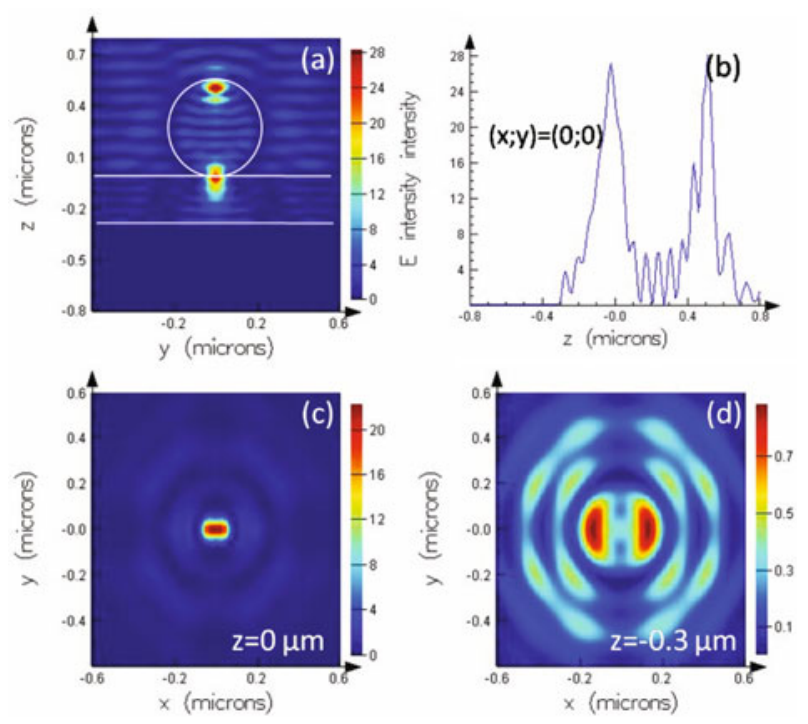

Figure 2. FDTD simulation of the intensity enhancement at the vicinity of $\mathrm{SiO}_{2}$ particles supported by a $300 \mathrm{~nm}$ $\mathrm{SiO}_{2}$ film (onto $\mathrm{Si}$ ). The side-view calculation (a) and the intensity profile along $z$ (b) reveal an enhancement exceeding $\times 25$ at the top and bottom of the particle. The $x-y$ intensity profiles at the $\mathrm{air} / \mathrm{SiO}_{2}$ surface (c) and the $\mathrm{SiO}_{2} / \mathrm{Si}$ interface (d) show laser hot spots. While the nanojet vanishes in $\sim 100 \mathrm{~nm}$ in the film (see (a) and (b)), a local contrast remains visible at the Si surface (d).

The 3D-domain was modelled using the real permittivity for silica $(n=1.45)$ and complex permittivity for silicon at $193 \mathrm{~nm}(n=0.847+\mathrm{i} 2.73)$. The structure is surrounded by the air index $(n=1)$. At the input face to the domain, a linearly polarized (along $\mathrm{x}$ ) transverse electromagnetic wave of unit intensity is applied, propagating normal to the substrate (along $\mathrm{z}$ ). The particle interface is defined at $z=0$. To minimize calculation times, the numerical simulations are performed only on one quarter of the total domain by setting symmetric (/xz) and anti-symmetric (/yz) boundary conditions. All other faces of the domain use a perfectly absorbing boundary condition [14]. The program was tested first on a single $\mathrm{SiO}_{2}$ particle in vacuum to make sure the solution agrees with the analytical solution of the generalized Mie theory. Then, we perform the calculations taking into account the presence of the substrate interfaces. Intensity enhancements are calculated as $|E|^{2}$ where $E$ is the laser electric field.

\subsection{Results and discussions}

In the experiments, we produce photonic nanojets with transparent silica microspheres similar to those we used in our previous work to laser fabricate porous alumina membranes [7]. The figure 2 shows the geometrical characteristics of the photonic nanojets generated with $500 \mathrm{~nm}$ diameter $\mathrm{SiO}_{2}$ particles when $193 \mathrm{~nm}$ wavelenth light is used. Our FDTD program takes into account multiple reflexions at the interfaces - assuming a sphere deposited onto a $300 \mathrm{~nm}$ thick $\mathrm{SiO}_{2}$ film on $\mathrm{Si}$. The yz view shows an enhancement of the laser intensity that exceeds $\times 25$ over $\cong 100 \mathrm{~nm}$ along the propagation direction. Reflection at the $\mathrm{SiO}_{2} / \mathrm{Si}$ interface is at the origin of a retro-propagating wave. As seen on figures 2(a) and 2(b), the sphere microlens refocus the retro-reflected light at the entrance of the particle with similar peak intensity as that produced at the substrate surface. The retro-wave also interferes with the incoming wave producing a standing interference pattern with $\lambda / 2 \mathrm{n}$ periodicity.

Figure 3 shows scanning electron microscopy (SEM) images of substrates similar to those simulated. To get a better insight of the sample affects, these are previously cleaved and observed tilted by 75 degrees to evaluate the surface morphology and to characterize in-bulk material modifications. The 

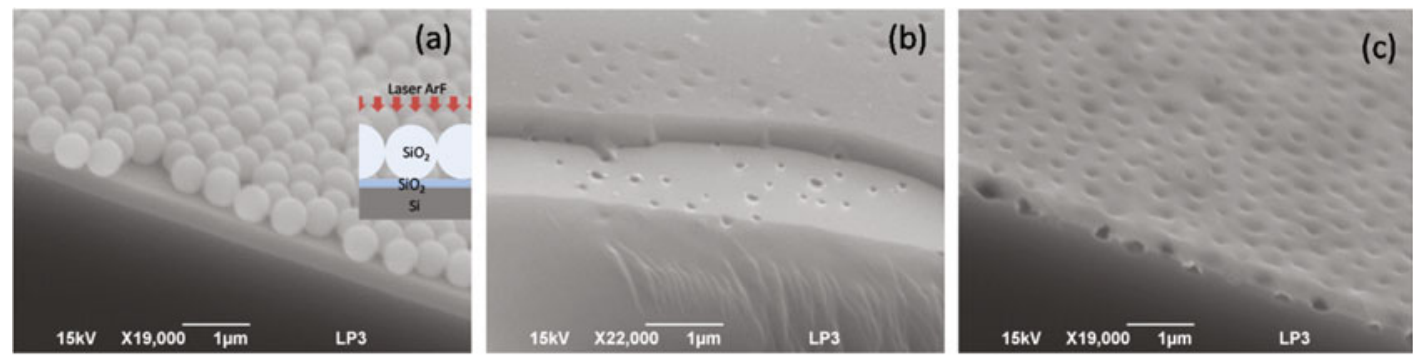

Figure 3. SEM side-view of $300 \mathrm{~nm}$ thick oxide films. (a) $500 \mathrm{~nm}$ diameter $\mathrm{SiO} 2$ spheres are deposited on the substrate. Then they are illuminated by a single nanosecond UV laser pulse $\left(\lambda_{\text {las }}=193 \mathrm{~nm}\right.$ ). (b) When the laser fluence is set at $F=700 \mathrm{mJcm}^{-2}$, the holes are produced in the film at the vicinity of the sphere and at the $\mathrm{SiO}_{2} / \mathrm{Si}$ interface. (c) Increasing the laser fluence to $F=900 \mathrm{mJcm}^{-2}$, strong damages are observed at the $\mathrm{SiO}_{2} / \mathrm{Si}$ interface but the film is not ejected.

figure 3(a) reveals the coverage of the oxidized substrate by our microspheres before illumination. The assembled monolayer exhibits defects. A more detailed study on the self-assembling technique would guarantee fewer defects but many domains larger than $50 \mu \mathrm{m}^{2}$ are manufactured with our current monolayer preparation approach which is enough for proof-of-concept experiments. By analyzing the surfaces with SEM imaging, we start to observe craters can be initiated at a laser fluence $F_{t h}$ of $\cong 650 \mathrm{mJcm}^{-2}$ ) for $100,300,500$ and $1000 \mathrm{~nm}$ thick oxide films on silicon.

Figure 1(c) (-top) shows the $100 \mathrm{~nm} \mathrm{SiO}_{2}$ films are fully drilled by the nanojet at typical fluence of $800 \mathrm{~mJ} \mathrm{~cm}^{-2}$. The size of craters is on the order of $150 \mathrm{~nm}$. When increasing the laser fluence to $1 \mathrm{Jcm}^{-2}$, just below the threshold for catastrophic damages, the lateral dimension of craters reaches $\cong 300 \mathrm{~nm}$ (Fig. 1(c)-bottom). As we expected, the fluence threshold for film removal increases with the film thickness since the local intensity on Si decreases and the amount of material to be removed increases. The figures 3(b) and 3(c) illustrate how $300 \mathrm{~nm}$ thick films are affected when illuminated in the same fluence range. Interestingly, cleaving the sample for imaging preparation has induced the breaking of the 300-nm thick oxide film (see Fig. 3(c)). Then the image exhibits directly the surface modifications of the Si substrate after illumination. While the film is not fully ablated, small nanocraters are found at both interfaces evidencing the peak intensity at the $\mathrm{Air} / \mathrm{SiO}_{2}$ interface is enough for direct ablation of the oxide. Also craters at the $\mathrm{SiO}_{2} / \mathrm{Si}$ interface show the residual light reaching the substrate exceeds the ablation threshold of Si. Difference of material response from $100 \mathrm{~nm}$ to $300 \mathrm{~nm}$ is in good accordance with the propagation length of the photonic nanojets simulated by FDTD (fig. 2) since we predicted the local intensity peaks $50 \mathrm{~nm}$ below the surface and is spread over $\sim 100 \mathrm{~nm}$. For this reason we cannot expect complete direct ablation of the film for the thicker films. Also the interaction at the $\mathrm{Si}$ interface is not strong enough to eject the film on top of it.

\section{CONCLUSION}

In conclusion, we demonstrate that 2D-periodic laser imprinting of transparent film onto absorbing substrate is feasible by combining UV light with monolayers of $500 \mathrm{~nm}$ diameter $\mathrm{SiO}_{2}$ spheres. The short propagation length of the photonic nanojet (fig. 2) makes only possible to drill films with thickness up to $100 \mathrm{~nm}$ in the fluence range we have tested $\left(\sim 1 \mathrm{~J} \mathrm{~cm}^{-2}\right)$. Working with thicker films, due to the local intensity enhancement, the oxide surface exhibits craters. At the Si surface, the intensity remains large enough to initiate ablation so the film is separated from the surface but without being popped off. The interaction between multilayer structures, particles and lasers is an interesting issue to be discussed since they are envisioned for nanofabrication techniques [7]. The microspheres we consider in this work are today routinely available and produce a wide variety of features depending on the illumination 
parameters and substrate preparation (thin film deposition). For these reasons, the approach opens routes to fabricating nanomaterials.

\section{References}

[1] Ed. Jim Greer, Anatoli Korkin, Jan K. Labanowsk, Nano and Giga Challenges in Microelectronics, (Elsevier Editions, 2003).

[2] P. N. Prasad, Nanophotonics (Wiley-IEEE, 2004).

[3] Y. Zhou, M. H. Hong, F. Fuh, L. Lu, B. S. Luk’yanchuck, Z. B. Wang, L. P. Shi, and T. C. Chong, App. Phys. Lett. 88, 023110 (2006).

[4] W. Cai and R. Piestun, Appl. Phys. Lett. 88, 111112 (2006).

[5] D. Grojo, A. Cros, P. Delaporte, and M. Sentis, Appl. Surf. Sci. 253, 8309 (2007).

[6] E. McLeod and C. B. Arnold, Nature Nanotech. 3, 413 (2008).

[7] A. Pereira, D. Grojo, M. Chaker, P. Delaporte, D. Guay, and M. Sentis, Small 4, 572 (2008).

[8] G. Langer, D. Brodoceanu, and D. Bauerle, Appl. Phys. Lett. 89, 261104 (2006).

[9] D. Grojo, L. Charmasson, A. Pereira, M. Sentis, and Ph. Delaporte, J. Nanosci. Nanotechnol. Submitted (2010)

[10] S.-M. Yang, S. Guy Jang, D.-G. Choi, S. Kim, and K. Y. Hyung, Small 2, 458 (2006).

[11] N. D. Denkov, O. D. Velev, P. A. Kralchevsky, I. I. B., H. Yoshimura, and K. Nagayama, Langmuir 8, 3183 (1992).

[12] A. S. Dimitrov and K. Nagayama, Langmuir 12, 1303 (1996).

[13] D. Grojo, P. Delaporte, M. Sentis, O. H. Pakarinen, and A. S. Foster, Appl. Phys. Lett. 92, 033108 (2008).

[14] G. Mur, IEEE Trans. Electromagn. C EMC-23, 377382 (1981). 